\title{
Can sequential coagulation monitoring predict major hemorrhage in extremely low birth weight infants?
}

Margarita Thanhaeuser ${ }^{1}$, Christoph Binder ${ }^{1}$, Ulla Derhaschnig, ${ }^{2,3}$, Bernd Jilma ${ }^{2}$, Margit KornsteinerKrenn ${ }^{1}$, Mercedes Huber-Dangl${ }^{1}$, Alexandra Kreissl ${ }^{1}$, Angelika Berger ${ }^{1}$, Nadja Haiden²

1 Department of Pediatrics, Division of Neonatology, Pediatric Intensive Care Medicine \& Neuropediatrics, Medical University of Vienna

2 Department of Clinical Pharmacology, Medical University of Vienna, Austria

3 Department of Emergency Medicine, Medical University of Vienna, Austria

\section{Background}

Bleeds such as intraventricular (IVH) and pulmonary haemorrhage $(\mathrm{PH})$ are life-threatening events in extremely low birth weight (ELBW) infants. Serial coagulation monitoring by measuring the international normalized ratio (INR) with small volume samples might facilitate early diagnosis and possibly prevent major bleeds.

\section{Patients \& Methods}

This was a prospective longitudinal study performed in ELBWinfants, who received serial INR monitoring by point of care testing during their first 30 days of life. The primary objective was to explore whether INR monitoring could predict major bleeding events (IVH, PH). Secondary objectives were mortality and feasibility in this patient population.

\section{Results}

127 ELBW-infants were stratified into a bleeding and a nonbleeding group. Any bleeding (IVH 24\%, PH 9\%) occurred in $31 \%$ $(39 / 127)$. Infants in the bleeding group were 4 days younger at birth $(p=0.05)$ and had a substantially higher mortality rate of $26 \%$ versus $5 \%$ in controls $(p=0.005)$. Median INR during the first three days before a bleeding event was 1.55 (95\% Cl: 1.39 1.74) compared to the control group with $1.45(95 \% \mathrm{Cl}$ : 1.44 $1.58 ; \mathrm{p}=0.81)$. Platelet counts were significantly lower in the bleeding group on the $3 \mathrm{rd}$ day and during the 2 nd-4th week of life.

\begin{tabular}{|c|c|c|c|}
\hline Obstetric history of the mother & $\begin{array}{l}\text { No Bleeding } \\
(n=88)\end{array}$ & $\begin{array}{l}\text { Bleeding } \\
(n=39)\end{array}$ & $\mathbf{p}$ \\
\hline & n (\%) & $\mathrm{n}(\%)$ & \\
\hline Caesarean section & $80(91 \%)$ & 31 (79\%) & 0.07 \\
\hline Preeclampsia & $3(3 \%)$ & $4(10 \%)$ & 0.12 \\
\hline Gestosis & $1(1 \%)$ & $1(4 \%)$ & 0.55 \\
\hline HELLP-Syndrome & $3(3 \%)$ & $4(10 \%)$ & 0.12 \\
\hline Preterm rupture of membranes & $30(34 \%)$ & $14(36 \%)$ & 0.84 \\
\hline Chorioamnionitis & $50(57 \%)$ & $14(36 \%)$ & 0.03 \\
\hline Antibiotic therapy (mother) & $58(66 \%)$ & $17(44 \%)$ & 0.02 \\
\hline Antihypertensive therapy (mother) & $8(9 \%)$ & $5(13 \%)$ & 0.52 \\
\hline Antenatal steroids (complete course) & $46(52 \%)$ & $21(54 \%)$ & 0.16 \\
\hline $\begin{array}{l}\text { Antenatal steroids (incomplete } \\
\text { course) }\end{array}$ & $40(45 \%)$ & $12(31 \%)$ & 0.12 \\
\hline \multirow[t]{2}{*}{ Demographic \& outcome data } & $\begin{array}{l}\text { No Bleeding } \\
(\mathbf{n}=\mathbf{8 8})\end{array}$ & $\begin{array}{l}\text { Bleeding } \\
(n=39)\end{array}$ & $\mathbf{p}$ \\
\hline & median (range) & median (range) & \\
\hline Birth weight, $\mathrm{g}$ & 751 (430-995) & $680(420-985)$ & 0.13 \\
\hline Gestational age & $25+6(23+1-33+4)$ & $25+2(23+2-29+0)$ & 0.05 \\
\hline \multirow[t]{2}{*}{ Days in the NICU } & $87(10-212)$ & $84(3-181)$ & 0.42 \\
\hline & n (\%) & n (\%) & \\
\hline Male & $49(56 \%)$ & $25(64 \%)$ & 0.37 \\
\hline Deceased & $4(5 \%)$ & $10(26 \%)$ & 0.005 \\
\hline SGA & $22(25 \%)$ & $10(26 \%)$ & 0.93 \\
\hline NEC & $8(9 \%)$ & $4(10 \%)$ & 0.84 \\
\hline NEC surgery & $1(1 \%)$ & $2(5 \%)$ & 0.17 \\
\hline PDA & $63(72 \%)$ & $35(90 \%)$ & 0.025 \\
\hline BPD & $21(24 \%)$ & $8(21 \%)$ & 0.68 \\
\hline ROP > grade 3 & $8(9 \%)$ & $2(5 \%)$ & 0.44 \\
\hline
\end{tabular}

\begin{tabular}{|l|c|c|c|}
\hline INR Screening & $\begin{array}{c}\text { No Bleeding } \\
\mathbf{( n = 8 8 )}\end{array}$ & $\begin{array}{c}\text { Bleeding } \\
\mathbf{( n = 3 9 )}\end{array}$ & $\mathbf{p}$ \\
\hline Day of life & median (range) & median (range) & \\
\hline $1-3$ & $1.45(1.1-2.1)$ & $1.55(1.0-2.0)$ & 0.81 \\
\hline $4-7$ & $1.38(1.9-3.0)$ & $1.33(1.0-2.0)$ & 0.77 \\
\hline $8-14$ & $1.2(0.9-2.9)$ & $1.2(0.95-2.05)$ & 0.82 \\
\hline $15-21$ & $1.13(1.0-3.0)$ & $1.1(0.9-1.5)$ & 0.91 \\
\hline $22-30$ & $1.1(0.9-1.95)$ & $1.18(0.9-1.8)$ & 0.49 \\
\hline
\end{tabular}
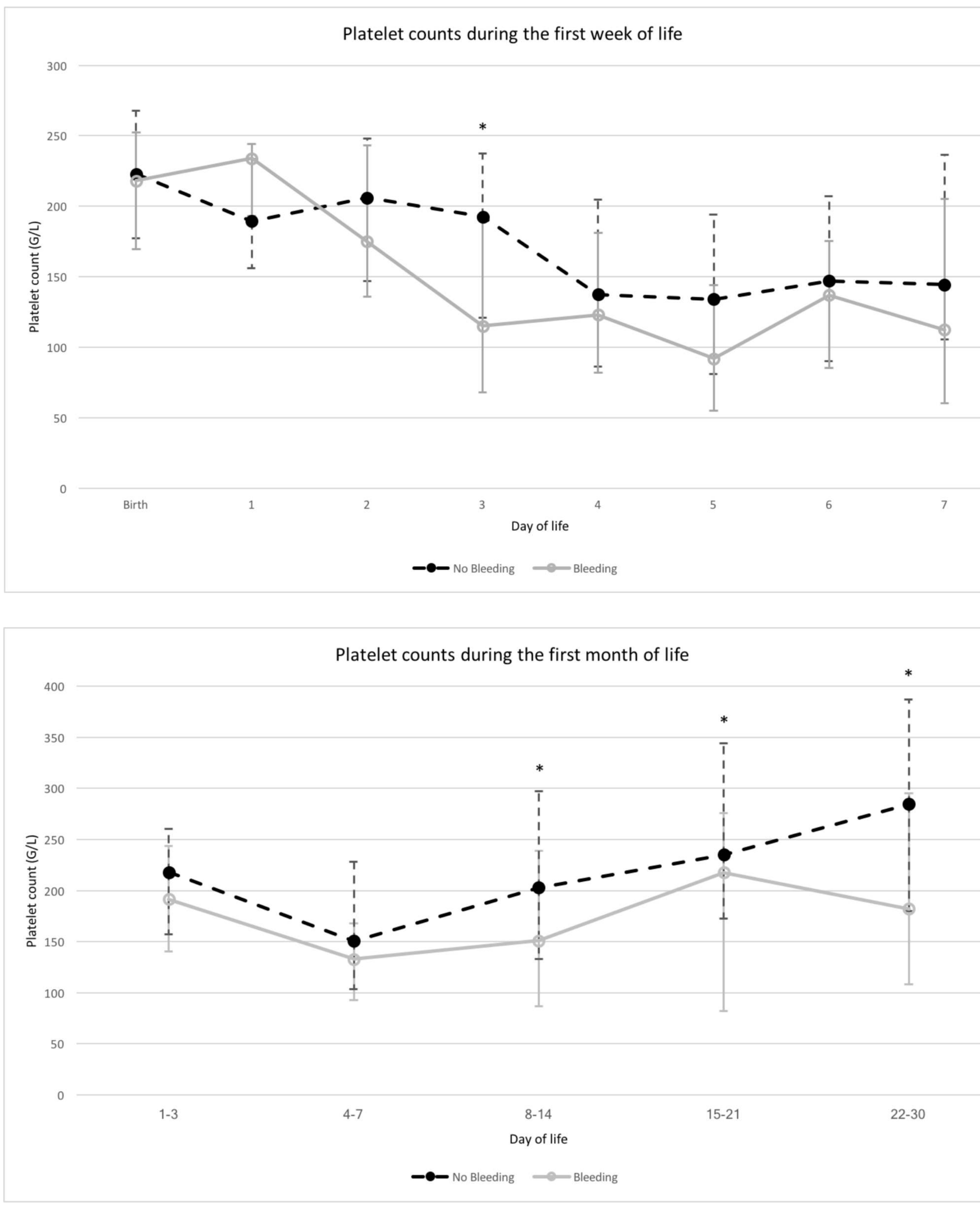

\section{Conclusion}

Serial coagulation monitoring by INR point of care testing is feasible in ELBW-infants but could not predict bleeding events. Further studies with daily monitoring of INR and platelet counts during the first days of life might more precisely detect a risk of major haemorrhage in ELBW-infants.

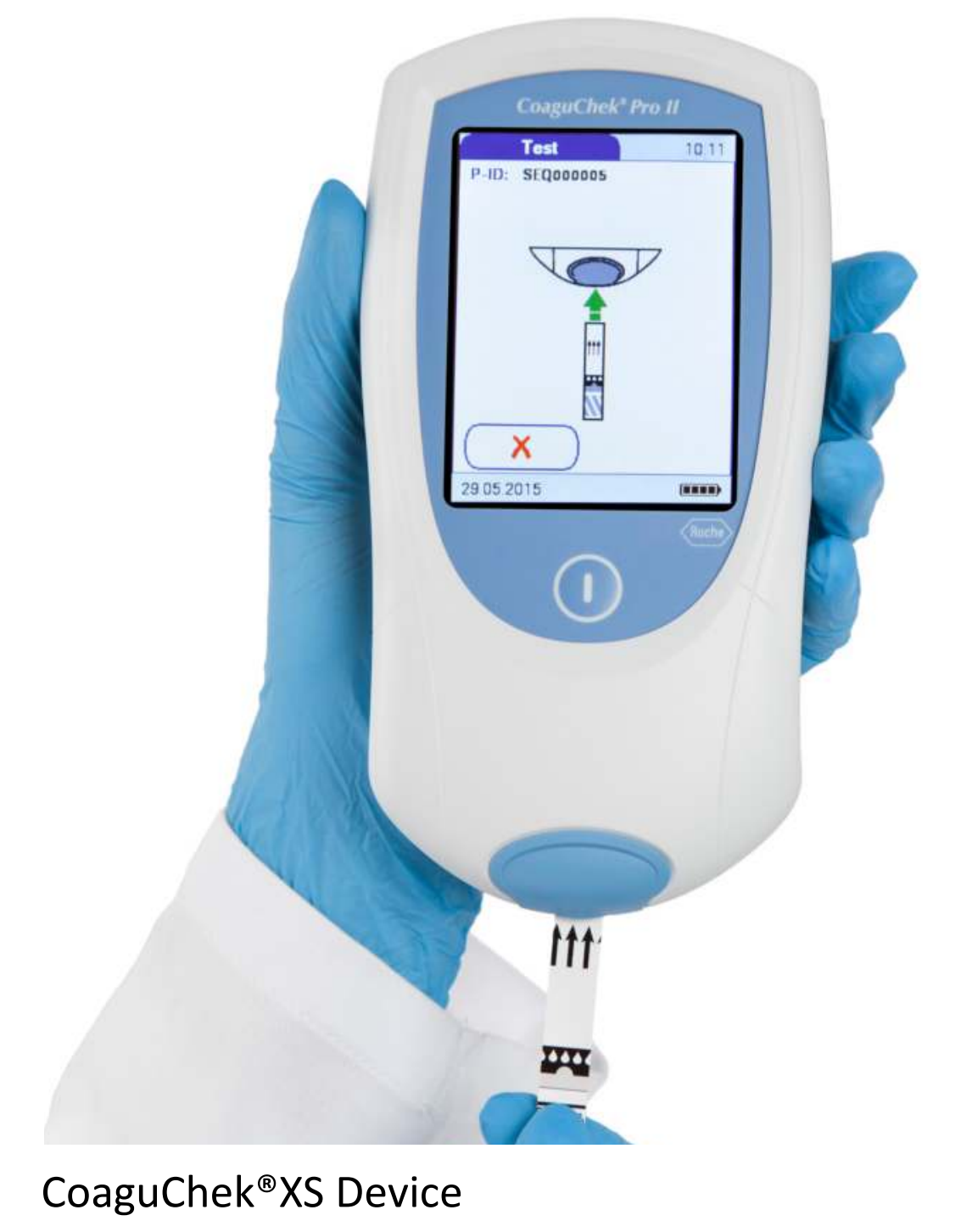

\section{References}

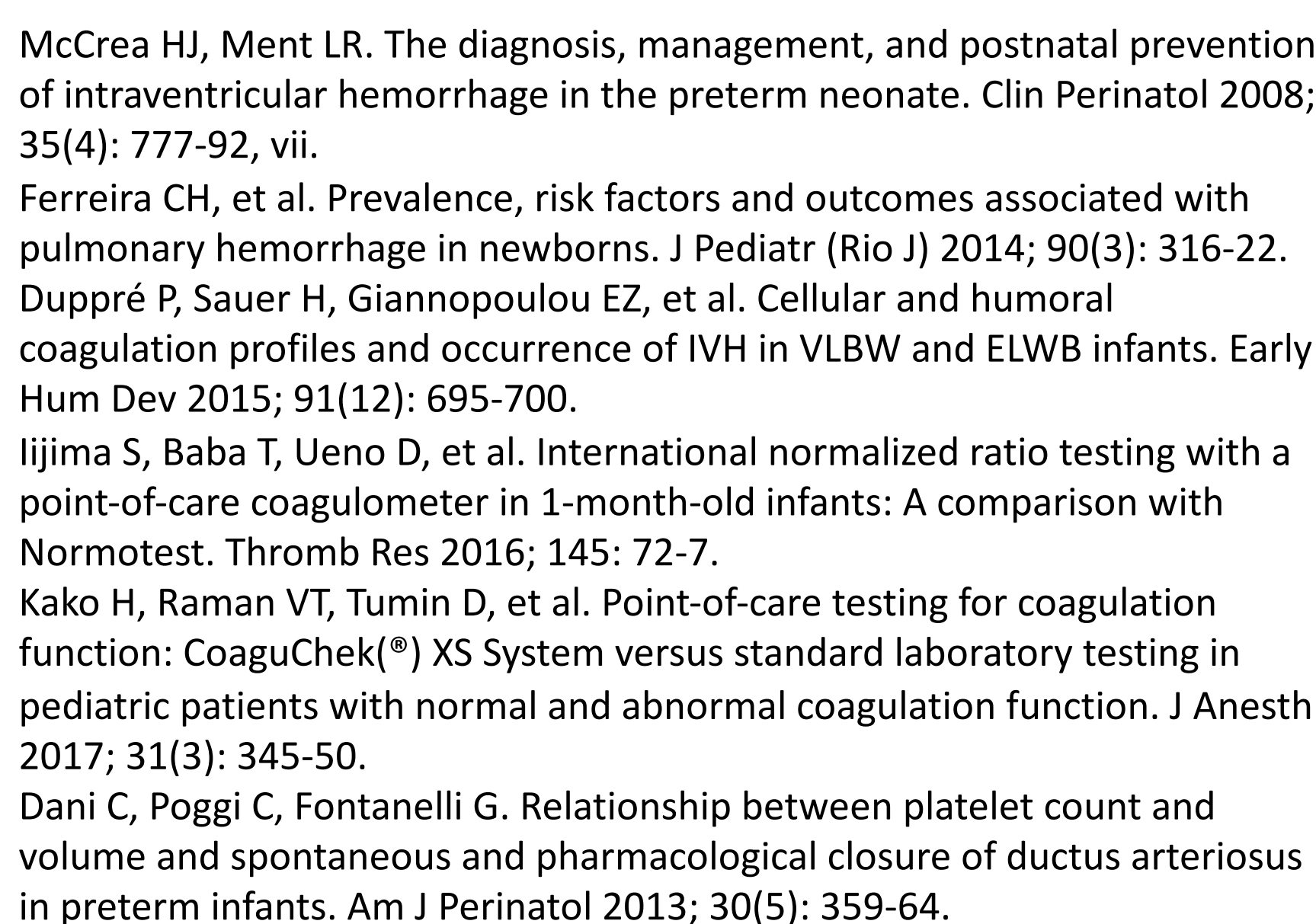

